\title{
Experimental lateral wall boundary layer behavior of a differentially rotating split-cylinder flow
}

\author{
J. O. Rodríguez-García* and J. Burguete ${ }^{\dagger}$ \\ Departamento de Física y Matemática Aplicada, Universidad de Navarra, P.O. Box 177, E-31008 Pamplona, Spain
}

(Received 31 August 2018; revised manuscript received 10 January 2019; published 19 February 2019)

\begin{abstract}
The cylindrical wall boundary layer of a closed cylinder split in two halves at the equator is studied experimentally. When these two parts rotate in exact corotation the internal flow is essentially in solid-body rotation at the angular velocity of both halves. When a slight difference between the rotation frequencies is established a secondary flow is created due to the differential rotation between both sides and restricted to the boundary layer. This behavior of the boundary layer is compared with theoretical and numerical results finding the "sandwich" structure of a Stewartson boundary layer. Time-dependent waves are observed near the cylindrical wall. Their behavior for different values of the control parameters are presented. Finally, a global recirculation mode is also found due to a symmetry-breaking induced between sides that appears because of a slight misalignment of the experimental setup, whose characteristics are compatible with the behavior of a precessing cylinder.
\end{abstract}

DOI: 10.1103/PhysRevE.99.023111

\section{INTRODUCTION}

Instabilities and transition to turbulence in rotating flows still engage much attention due to their fundamental and practical interest (e.g., see Ref. [1]). From natural flows (tornadoes, ocean currents) to industrial flows (industrial mixers, wingtip vortices, turbomachinery), rotation is present in many scales, most of the times ruling the dynamics of the system. These flows can be classified as open flows (e.g., see Refs. [2-5]) or confined flows (e.g., see Refs. [6-9]).

Regarding these confined problems, the instabilities created in a closed cylindrical container have been extensively studied. When the fluid is in a laminar regime, the flow created can generate coherent structures that have been analyzed numerically and experimentally $[10,11]$. In the turbulent regime, the global recirculation can destabilize, and a rich dynamics appears $[8,9,12,13]$. Apart from the interest as a fundamental problem of fluid mechanics, the presence of instabilities on these flows can affect other processes: these flows have been used experimentally to generate the dynamo action [14,15], and it has been observed that the presence of instabilities can significantly affect the threshold of this effect $[16,17]$. These instabilities can also affect the dynamics of inertial particles inside these flows $[18,19]$.

A differential rotation in a cylindrical geometry can trigger different instabilities where the boundary conditions play a decisive role [7,20]. But, when the background rotation is fast enough, the Coriolis restoring force tends to restrict secondary flows to the boundary layers [21-23]. Then the flow developed inside the container is basically in solid-body rotation at the mean rotation of the system.

\footnotetext{
*jrodriguez.65@alumni.unav.es

†javier@unav.es
}

Focusing on these fast-rotating systems with differential rotation Stewartson [24] studied, in the inviscous limit, the case of a closed cylinder where the cylindrical wall rotates slightly faster than the end walls. He found that the inner flow is in almost solid-body rotation, and only a weak meridional flow appears driving the flow from one end wall boundary layer (Ekman type) [25] to the other along the cylindrical wall boundary layer which has a "sandwich" structure with two parts (Stewartson boundary layer): the inner part whose thickness is $O\left(v^{1 / 4}\right)$ and the main outer part whose thickness is $O\left(v^{1 / 3}\right)$ being $v$ the kinematic viscosity of the considered fluid. Hocking [26] studied the problem of an infinite cylinder split in two where one side rotates slightly faster than the other. In this case, the meridional flow due to the Ekman pumping is neglected. Later, van Heijst [27] studied the finite case of the split cylinder with a differential rotation using a boundary layer analysis in the limit of very fast rotation velocity (inviscous problem) and very small differential rotation. Gutierrez-Castillo and Lopez [28] reproduced this analysis numerically in two dimensions (axisymmetric flows) and extended it for large but finite rotation velocities (nonlinear viscous problem) and larger differential rotations studying the stability of the main flow. Later this numerical analysis was extended in three dimensions [23] finding nonaxisymmetric instabilities. For this corotating split cylinder, the flow developed inside is in almost solid-body rotation at the average rotation rate, as in the Stewartson's problem, and a meridional flow appears driving fluid from one end wall to the other with the sandwich structure found in Ref. [24]. For a large enough differential rotation, instabilities can appear on the confluence of both boundary layers (end wall: Ekman type; lateral wall: Stewartson type) and propagate through the cylindrical wall creating periodic or quasiperiodic states [28].

The principal purpose of this work is the experimental study of this corotating split-cylinder configuration focusing on the cylindrical wall boundary layer for a large range of 
parameters. We have analyzed the dynamical behavior of the velocity components and characterized the different flow regions that can be distinguished on the cavity. Finally, we have studied a global recirculation triggered by a small symmetry breaking of the experimental setup and whose characteristics are similar to the recirculations present on precessing setups.

\section{EXPERIMENTAL SETUP}

The experimental setup consists of a horizontal hollow cylinder which is split in two halves at midlength (see Fig. 1). Lateral cylindrical surfaces are made of PMMA and bases are made of aluminum. The internal radius $R$ of the cylinder is fixed and equal to $5 \mathrm{~cm}$, but the internal length $L$ of each half can be modified using different aluminum bases. Methacrylate cylindrical walls have $8.5 \mathrm{~mm}$ of thickness and $0.05 \mathrm{~mm}$ of tolerance. Aluminum pieces have $0.1 \mathrm{~mm}$ of tolerance at maximum. A gap between halves is needed in order to avoid friction and deformation of semicylinders during rotation. This gap is fixed and equal to $0.2 \mathrm{~mm}$, and this is the only place where a mass interchange may happen between the inner and the outer parts of the cylinder. The split cylinder is enclosed in a prismatic cell of 121 filled by the working fluid. Shafts are moved by two independent servomotors of $750 \mathrm{~W}$ of power each one, allowing a rotation frequency of both halves in the range $f=0-100 \mathrm{~Hz}$. The velocity of the motors is controlled by two servo drives governed by two waveform function generators. The accuracy of the rotation velocity is $0.01 \%$ of the desired velocity. With this configuration, the halves of the split cylinder can rotate in corotation or in counter-rotation and their rotation velocity can be equal or not. The fluid used as working fluid is water at a controlled temperature. This temperature is taken into account in order to determine the viscosity.

The experimental velocity field developed inside the split cylinder is measured using a 1D LDV system $(514.5 \mathrm{~nm})$ with an ellipsoidal measurement volume of $1.7 \mathrm{~mm}$ of major axis and $90 \mu \mathrm{m}$ of minor axes. The LDV system allows the measurement of two components of the velocity field (axial $v_{z}$ and azimuthal $v_{\theta}$ ), which are measured in a horizontal plane

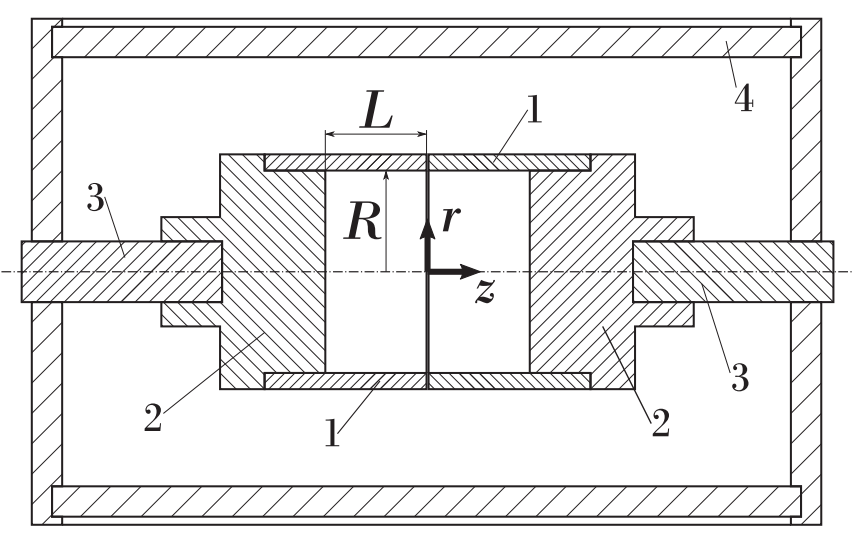

FIG. 1. Cross section of the split cylinder inside the cell (top view). Parts list: 1 . Methacrylate semicylinders; 2 . Aluminum bases; 3. Stainless steel shafts; 4. External walls of the cell. The axes correspond to the reference frame used, and $\theta$ is the rotation around z. $\theta=0$ corresponds to the plane above the symmetry axis. that contains the axis of the split cylinder $\left(\theta=0\right.$ or $\left.180^{\circ}\right)$. Due to the deflections of the laser beams crossing through different interfaces, optical corrections are taken into account to recover the correct value of the measured velocity. In order to optimize the use of this technique two types of particles have been used: silver-coated hollow glass spheres $(14 \mu \mathrm{m}$, $\left.\rho=1.65 \mathrm{~g} / \mathrm{cm}^{3}\right)$ and polyamide seed particles $(50 \mu \mathrm{m}, \rho=$ $1.03 \mathrm{~g} / \mathrm{cm}^{3}$ ). The first kind of particles have high reflectivity but a bad density matching, and the second kind of particles have high density matching but only $10 \%$ of the reflectivity of the first ones. The different experiments carried out using both kind of particles have shown no difference on the measurements, so glass spheres have been chosen over polyamide ones to improve reflectivity.

In this work, the split cylinder is rotating on corotation with different velocities in each half. To establish the velocities of the experiment a main rotation velocity $\Omega$ is selected in both halves and a differential rotation velocity $\omega(\omega<\Omega)$ is added or subtracted depending on the half $(\Omega \pm \omega)$. We start each experimental run from the fluid at rest. The desired velocity of each half is set in the drives and, then, the servomotors are switched on at the same time. They reach the required velocity in $0.2 \mathrm{~s}$. The measurements start $\sim 15 \mathrm{~min}$ after switching the motors on.

We have three experimental dimensionless control parameters that describe the state of the split cylinder, including its geometry. The first one is the Reynolds number defined using the main rotation velocity, $\operatorname{Re}=\frac{\Omega R^{2}}{v}$, where $v$ is the kinematic viscosity of the working fluid [29]. Re has been varied in the range $\operatorname{Re} \sim 4 \times 10^{3}-2 \times 10^{4}$. The second parameter is the Rossby number that establishes a relationship between the main and the differential angular velocities, Ro $=\frac{\omega}{\Omega}$. Ro has been varied in the range Ro $\sim 0-0.4$ where $\mathrm{Ro}=0$ represents the exact corotation case. For this $\mathrm{Ro}=0$ the split cylinder has reflection symmetry respect to a equatorial plane $(z=0)$. This symmetry is broken when a Ro $\neq 0$ is established. Finally, the geometrical parameter is the aspect ratio of the experimental volume, $\Gamma=\frac{2 L}{R}$, which has been fixed to 2 in this work.

\section{EXPERIMENTAL RESULTS}

For Ro $=0$ the expected behavior is just a flow in solidbody rotation at $\Omega$ with perfect reflection symmetry in the equatorial plane for the range of studied Re. When a Ro $\neq 0$ is set, then the flow keeps this solid-body rotation far from the cylindrical walls. In this second case, the boundary layer of the bases (Ekman type) and the boundary layer of the cylindrical wall (Stewartson type) [25] meet on the corners and an instability in the form of periodic or quasiperiodic states can appear and propagate from the faster corner to the slower corner depending on the Ro according to Refs. [23,28]. Hence, the zone near the cylindrical wall has to adapt its angular velocity from the solid-body rotation $(\Omega)$ to the wall rotation $(\Omega \pm \omega)$. In this situation, a weak axial velocity has to appear near the cylindrical wall driving the instability from the faster to the slower corner. In this work, we have studied only the cylindrical wall boundary layer and not the Ekman one at the end caps due to the difficulty of measuring the experimental velocity field near the bases. All results are presented 


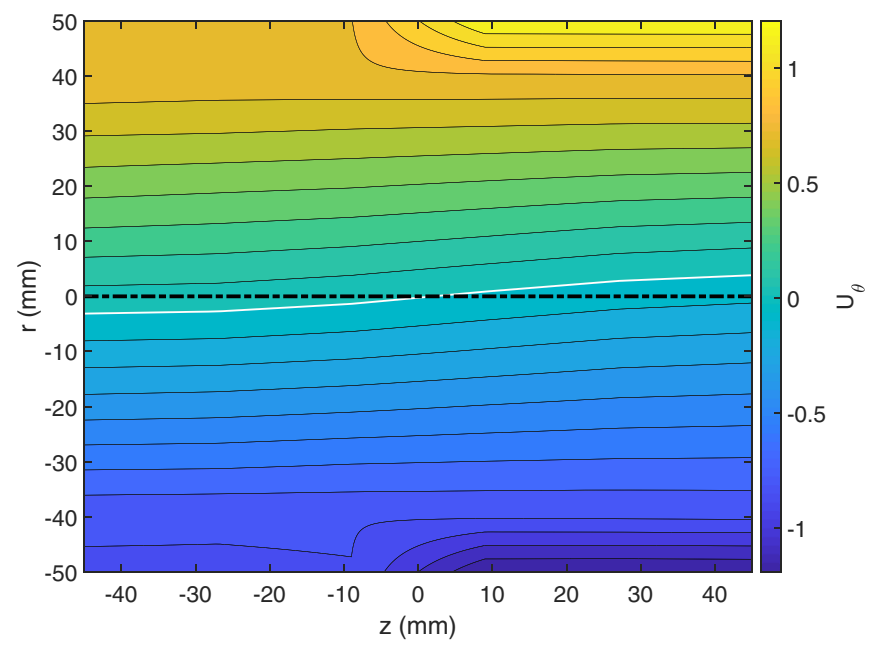

FIG. 2. Time-averaged dimensionless azimuthal velocity (interpolated field) in a horizontal plane (half-planes with $\theta=0^{\circ}$ and $180^{\circ}$ ) at a refined mesh of $91 \times 101$ points (one point each $\mathrm{mm}$ ) for $\mathrm{Re}=1.4 \times 10^{4}$ and $\mathrm{Ro}=0.2$. There are 25 linearly spaced contour levels in the range $U_{\theta} \in[-1.2,1.2]$, where a positive sign is used to mean that $U_{\theta}$ goes out of the plane, and a negative sign means that $U_{\theta}$ goes into the plane. The white thick curve corresponds to the contour level $U_{\theta}=0$.

nondimensionalized using the characteristic velocity $V=\Omega R$ (dimensionless velocities are denoted as $u$ ).

The time-averaged dimensionless azimuthal velocity $U_{\theta}(r, z)=\left\langle u_{\theta}(r, z, t)\right\rangle$ shown in Fig. 2 is obtained using the LDV system in a mesh of $6 \times 11(z \times r)$ equispaced points $(-45 \mathrm{~mm} \leqslant z \leqslant 45 \mathrm{~mm}$ and $-50 \mathrm{~mm} \leqslant r \leqslant 50 \mathrm{~mm})$. The time average, denoted as $\langle\cdot\rangle$, is computed for each point along the whole acquisition time (for the case considered, $120 \mathrm{~s}$ per point). The measurement is performed in a horizontal plane (composed of two half-planes with $\theta=0^{\circ}$ and $\theta=180^{\circ}$ ) for $\mathrm{Re}=1.4 \times 10^{4}$ and $\mathrm{Ro}=0.2$, and it is refined in a $91 \times 101$ points mesh before being plotted. Here the azimuthal flow presents a big internal bulk $(-40 \mathrm{~mm}<r<40 \mathrm{~mm})$, which is in almost solid-body rotation at $\Omega$ and a zone near the cylindrical wall $(-50 \mathrm{~mm} \leqslant r \leqslant-40 \mathrm{~mm}, 40 \mathrm{~mm} \leqslant r \leqslant$ $50 \mathrm{~mm}$ ) which adapts its angular velocity from that of the wall $\Omega \pm \omega$ to the solid-body rotation velocity $\Omega$ (this behavior can be compatible with the Basic State described in Refs. [23,28]). This azimuthal average flow is observed for the whole range of Re and Ro explored.

Nevertheless, the time-averaged azimuthal velocity contour lines presented in Fig. 2 are not parallel to the axis of rotation as would be expected in solid-body rotation. Instead, they are tilted breaking the expected reflection symmetry in the equatorial plane. This behavior will be discussed below and does not affect the boundary layer.

The mean flow is measured using a coarse mesh that cannot capture the complexity of the boundary layer. The region where the velocity changes from $\Omega$ to $\Omega \pm \omega$ seems to be of the order of $1 \mathrm{~cm}$ in Fig. 2 because of the acquisition mesh. To better characterize this boundary layer, the azimuthal velocity is measured along the radius for different axial positions with a very high spatial resolution (one measurement each $0.2 \mathrm{~mm}$ )

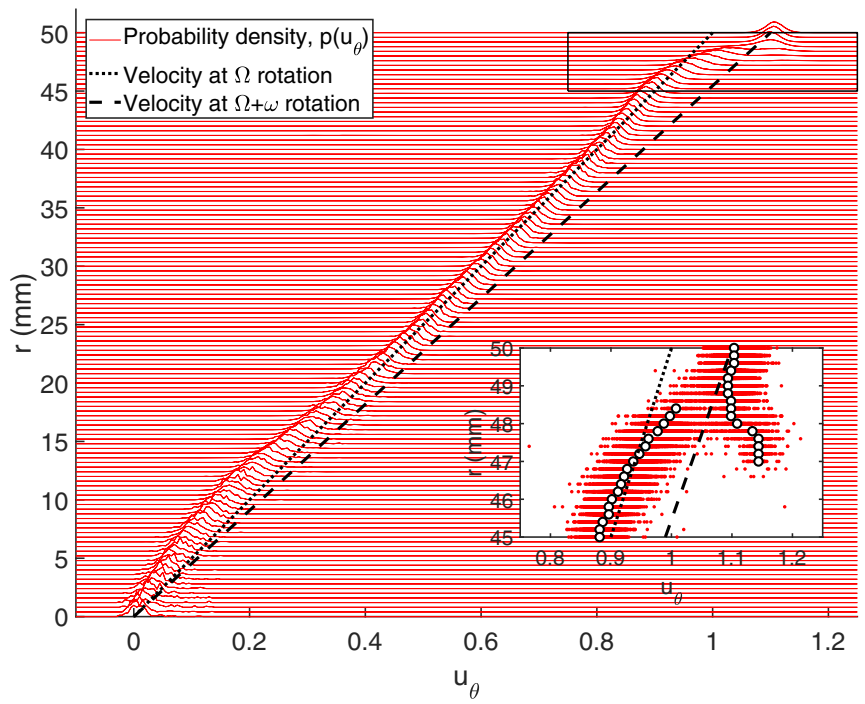

FIG. 3. PDFs of the dimensionless azimuthal velocity $u_{\theta}$ along a radius at $z=40 \mathrm{~mm}$ for $\mathrm{Re}=2 \times 10^{4}$ and $\mathrm{Ro}=0.1$. The dashed line represents the solid-body rotation $u_{\theta}=(1+\mathrm{Ro}) r / R$ with a rotation frequency $\Omega+\omega$, and the dotted line represents the solid-body rotation $u_{\theta}^{\text {solid }}(r)$. Inset: Detail of the velocity near the cylindrical wall (zoom of the marked rectangle) where the red points correspond to individual velocity measurements, and the hollow black points represent the means of the PDFs.

to really catch its behavior, using an acquisition time of $240 \mathrm{~s}$ per point.

We present an example of this behavior in Fig. 3 at $z=$ $40 \mathrm{~mm}$ for $\mathrm{Re}=2 \times 10^{4}$ and $\mathrm{Ro}=0.1$. In order to have a global view of the behavior of the velocity, the probability density functions (PDFs) of the dimensionless velocity are presented each $0.4 \mathrm{~mm}$. The expected behavior of the velocity consists of a central region where the fluid will rotate in almost solid-body rotation $u_{\theta}^{\text {solid }}(r)=r / R=(\Omega r) /(\Omega R)$ (dotted line), and an external shell, the boundary layer (whose thickness is $\ell_{B L}$ ), where the fluid adapts its velocity to that of the lateral wall $u_{\text {wall }}^{ \pm}=(1 \pm \operatorname{Ro})=(\Omega \pm \omega) R /(\Omega R)$ (dashed line) where the sign considered depends whether we are close to the fast $(+)$ or slow $(-)$ hemicylinder.

But in our experiment the velocity near the wall has a complex dynamics. Depending on the parameters, this behavior can be very weak, so it can be hardly detected using the PDFs. We present our data in a different way that enhances the presence of these weak processes in the region close to the wall. The inset details this behavior near the cylindrical wall where each red point represents a single velocity measurement. Using this approach, the velocity values appear concentrated on two regions around $u_{\theta}=0.95$ and $u_{\theta}=1.15$ for each value of $r$ in the region for $47 \mathrm{~mm}<r<48.5 \mathrm{~mm}$ separated by a region where no measurements are obtained between $1 \lesssim$ $u_{\theta} \lesssim 1.1$. Hollow black points represent the mean value of the velocity for each region. Connecting these hollow points we can distinguish two branches inside the boundary layer. One branch starts at $u_{\text {wall }}^{+}=(1+\mathrm{Ro})=[(\Omega+\omega) R] /(\Omega R)$, but then the velocity increases to a value larger than the wall velocity $u_{\text {wall }}^{+}$along a narrow zone $(\sim 2 \mathrm{~mm})$ and disappears for $r<47 \mathrm{~mm}$. The other branch adapts rapidly its velocity 
(a)

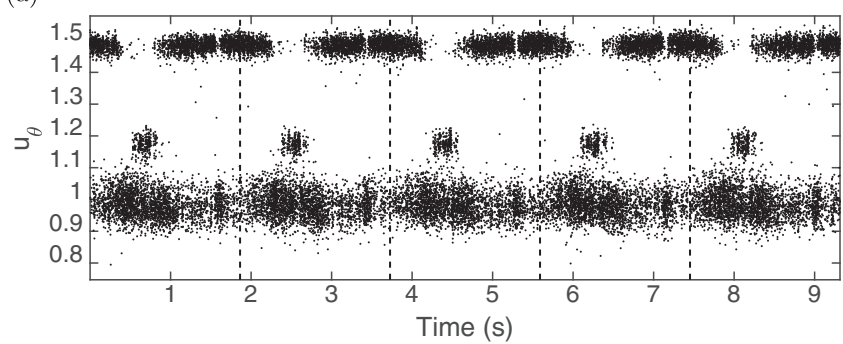

(b)

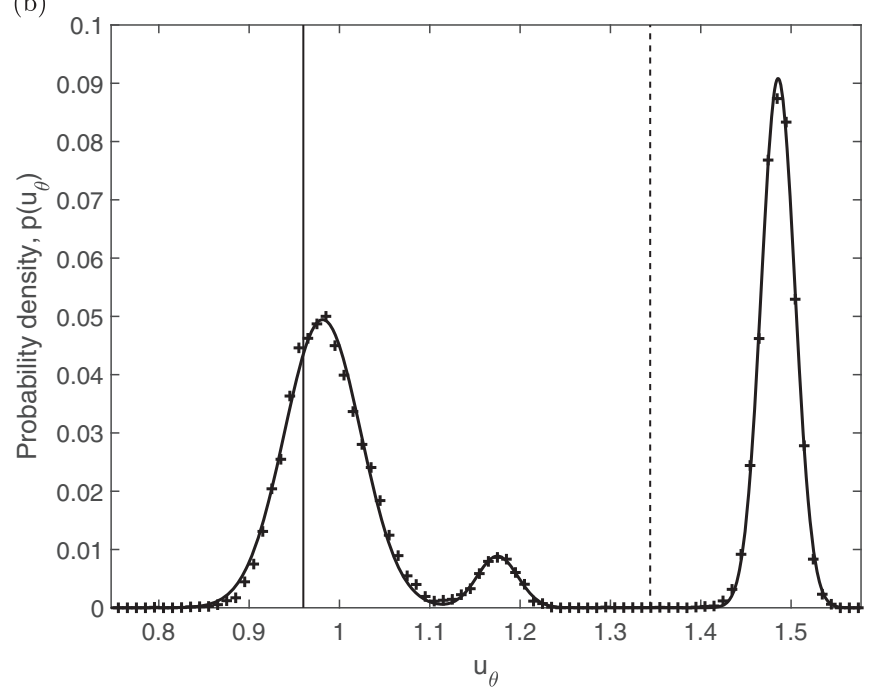

FIG. 4. Azimuthal velocity at $z=40 \mathrm{~mm}$ and $r=48 \mathrm{~mm}$ for $\operatorname{Re}=6 \times 10^{3}$ and Ro $=0.4$. (a) Azimuthal velocity folded in time and represented along five periods (dashed lines) using the period $2 \pi /(\Omega+\omega)$. (b) PDF of the experimental dimensionless azimuthal velocity (crosses), Gaussians fitting (solid curve), solid-body rotation at $u_{\theta}^{\text {solid }}\left(r_{48}\right)$ (vertical solid line), and solid-body rotation at $(1+$ $\mathrm{Ro})\left(r_{48} / R\right)$ (vertical dashed line).

from the faster rotation velocity $(\Omega+\omega)$ to the main angular velocity $(\Omega)$ and behaves like a solid-body in rotation everywhere but near the axis where the velocity is smaller than the solid-body rotation velocity $u_{\theta}^{\text {solid }}(r)$. It is convenient to notice that, due to the size of the measurement volume in the radial direction $(1.7 \mathrm{~mm})$, the experimental velocity obtained in each measurement point is affected by the value of the velocities from neighbor points increasing its standard deviation (i.e., a convolution between the real velocity and the ellipsoidal laser intensity distribution). Measurements performed for Fig. 3 included spurious data that correspond to reflections of the laser beams that cross again outside the cylinder in the outer water volume. These data, with a null velocity, have been removed from the series before the analysis.

From now on, we will focus on the flow behavior in the boundary layer near the cylindrical wall. This flow is studied measuring the azimuthal velocity on six equispaced points along the axial direction (from $z=-40 \mathrm{~mm}$ to $z=40 \mathrm{~mm}$ ) at a fixed radius $\left(r=48 \mathrm{~mm}\right.$, from now on referred as $\left.r_{48}\right)$ for the Re and Ro considered, and using the LDV system with an acquisition time of $300 \mathrm{~s}$ per point. In these points, it can be observed that the azimuthal velocity has, in general, a complex behavior, similar to the one shown in Fig. 4. In
Fig. 4(a) the azimuthal velocity series for $\operatorname{Re}=6 \times 10^{3}$ and Ro $=0.4$ at $z=40 \mathrm{~mm}$ and $r=48 \mathrm{~mm}$ presents different jumps among three different velocities. If we compare this behavior with the one presented on Fig. 3 for $r=48 \mathrm{~mm}$ we observe that now, for these parameters, we have three states that correspond to the previous two branches. The slowest branch is the one that connects the fast-spinning wall to the inner solid rotation flow this state being characterized by $u_{\theta}=48 / 50=0.96 \simeq 1$, and the fast branch where the fluid jumps between two states, $u_{\theta} \simeq 1.18$ and 1.48 . These jumps appear with a period equal to the one of the $\Omega+\omega$ frequency, as we are close to the fast wall [see Fig. 4(a) where the original experimental series taken along $300 \mathrm{~s}$ has been zoomed in folding the whole series along five periods $2 \pi /(\Omega+\omega)]$.

The velocity on the fast branch jumps between two values that are below and over the wall velocity [in Fig. 4, the wall velocity is $\left.u_{\text {wall }}^{+}=(1+\mathrm{Ro})=1.4\right]$. So in the reference frame of the moving wall the flow moves in both directions in the azimuthal direction, this result being compatible with a vortex or a roll. This structure is traveling near the wall and crosses through the measurement point at specific times giving the temporal behavior of the flow presented in Fig. 4.

The region where a coexistence of the two branches exists is $1.5 \mathrm{~mm}$ long (see Fig. 3, inset). Although these results point to the presence of both states at the same radial locations, we would like to recall that the LDA volume is $1.7 \mathrm{~mm}$ long, and so the presence of each one of these branches will be expanded radially because of the experimental technique. These results are compatible with an inner branch ending around $r \simeq 47.7 \mathrm{~mm}$ where the boundary layer branch starts, and then $\ell_{B L} \simeq 2.2 \mathrm{~mm}$.

It is expected that the behavior and dynamics of these two regions, the inner solid-body rotation and the boundary layer, will depend on the experimental parameters Re and Ro. In particular, we are interested on the characterization of the inner solid rotation body when the Coriolis force is increased (large Ro) compared to the exact corotation regime. Concerning the dynamics of the boundary layer, we would like to analyze the effect of the viscous forces ( $\mathrm{Re})$, and whether we can detect the instability threshold of this behavior. These two processes will be determined by the evolution as a function of $\mathrm{Re}$ and Ro of the corresponding PDFs [see Fig. 4(b)]. The experimental data can be described, as expected, by a combination of Gaussians,

$$
\operatorname{PDF}\left(u_{\theta}\right)=\sum_{i=1}^{N_{G}} a_{i} \frac{1}{s_{i} \sqrt{2 \pi}} \exp \left[\frac{-\left(u_{\theta}-\bar{u}_{\theta}^{i}\right)^{2}}{2 s_{i}^{2}}\right],
$$

where $N_{G}$ represents the number of Gaussians used to describe the data. $\bar{u}_{\theta}^{i}, s_{i}$, and $a_{i}$ are, respectively, the center, the standard deviation, and the relative weight of each normal distribution (where $\sum_{i=1}^{N_{G}} a_{i}=1$ ). For the case shown in Fig. 4(b) the PDF is described by the combination of $N_{G}=3$ different Gaussians (presented as a solid curve). We will focus on the evolution of the mean value $\bar{u}_{\theta}^{i}$ of each Gaussian together with its standard deviation $s_{i}$ (that will determine the corresponding error bar), but avoiding the relative weights $a_{i}$ (see Figs. 5-7). The relative weight of each Gaussian $a_{i}$ does not represent a correct parameter to characterize the instability, as it depends on how many tracers are trapped in these states. 


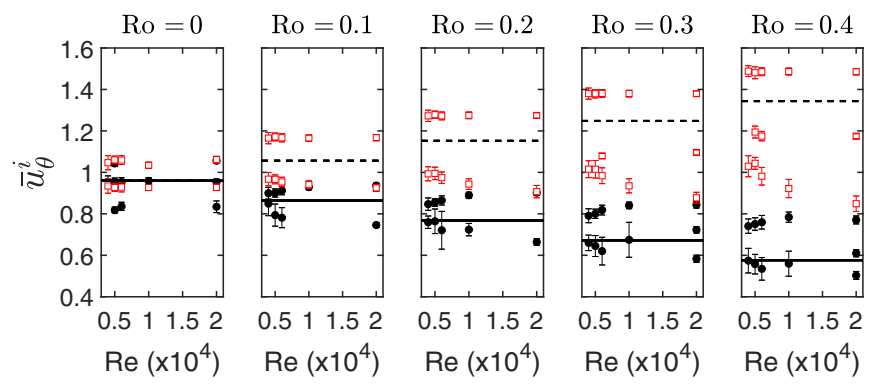

FIG. 5. Dimensionless azimuthal velocities $\bar{u}_{\theta}^{i}$ vs Re for different Ro. Black points represent the velocities at $r=48 \mathrm{~mm}$ and $z=$ $-40 \mathrm{~mm}$, and hollow red squares represent the velocities at $r=$ $48 \mathrm{~mm}$ and $z=40 \mathrm{~mm}$. The solid line represents the solid-body rotation at $(1-\mathrm{Ro})\left(r_{48} / R\right)$, and the dashed line represents the solidbody rotation at $(1+\mathrm{Ro})\left(r_{48} / R\right)$.

We can compare, for example, the evolution of these branches when the Re is increased for two points close to both cylinder ends. Figure 5 shows the behavior of the dimensionless azimuthal velocities $\bar{u}_{\theta}^{i}$ as a function of Re for different Ro at $z=-40 \mathrm{~mm}$ (solid black points) and $z=40 \mathrm{~mm}$ (hollow red squares). When Ro $=0$ (left panel) the flow is in solid rotation with a velocity that corresponds to $u_{\theta}\left(r_{48}\right)=r_{48} / R=$ 0.96 (solid thick line). Please note that $u_{\theta}=1$ corresponds to the lateral wall velocity $\Omega R$. Only Gaussians with a weight $a_{i}$ larger than $\sim 5 \%$ are retained $\left(a_{i}>0.05\right)$. When the Rossby number Ro is increased, the branches discussed above separate and both behaviors can be distinguished: a branch moving faster (respectively slower) than the wall when we are close to the fast (respectively slow) cylinder, and the other branch corresponds to the solid-rotation volume, where the Gaussians of these solid-rotation states for both halves converge to a value slightly below 1 when the Re number is increased. In both (fast and slow ends) cases, the velocity presents two states that separate when $\operatorname{Re}$ and Ro increase. For Ro $\geqslant$ 0.3 a third state could appear in between depending on the Re. On the faster side, one of these states has an angular velocity which is higher than the solid-body angular velocity at $\Omega+\omega$ for $r=48 \mathrm{~mm}$ [and even faster than the rotation of the cylindrical wall $u_{\theta}\left(r_{48}\right)>(1+$ Ro $\left.)\right]$ corresponding to the fastest branch of the boundary layer. Although it is clear that those branches separate when Re increases for Ro $>0$, we cannot define a threshold value. This threshold, if any, should be below our minimum achievable experimental Reynolds number, $\operatorname{Re}=4 \times 10^{3}$.

The evolution of these two branches also depends on the axial position of the measuring volume. Figure 6 represents the behavior of the dimensionless azimuthal velocities $\bar{u}_{\theta}^{i}$ as a function of Ro at the six z-locations for $\mathrm{Re}=4 \times 10^{3}$ (top panels) and for $\mathrm{Re}=2 \times 10^{4}$ (bottom panels). In this figure, it can be seen a state of the velocity in the slow cylinder $(z<0)$ which is smaller than the solid-body angular velocity at $\Omega-\omega, u_{\theta}\left(r_{48}\right)=(1-\mathrm{Ro}) r_{48} / R$. Meanwhile, in the fast cylinder $(z>0)$, there is the state which is faster than the solid-body rotation velocity at $\Omega+\omega, u_{\theta}\left(r_{48}\right)=(1+$ Ro) $r_{48} / R$. These two behaviors correspond to the branches that connect with the wall velocities, and we can observe that

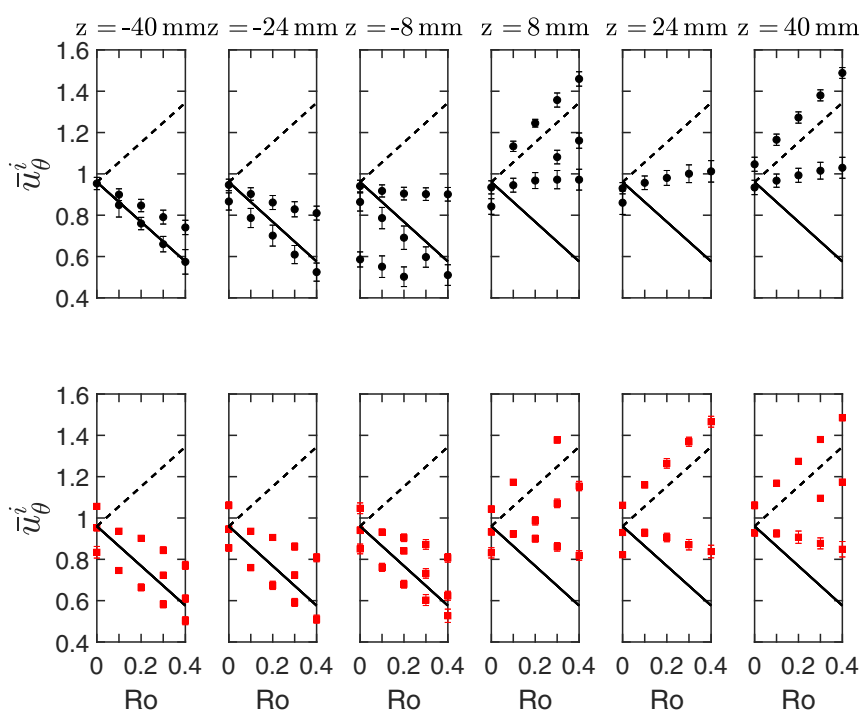

FIG. 6. Dimensionless azimuthal velocities $\bar{u}_{\theta}^{i}$ vs Ro along the axial direction at $r=48 \mathrm{~mm}$ for $\operatorname{Re}=4 \times 10^{3}$ (top panels) and $\mathrm{Re}=2 \times 10^{4}$ (bottom panels). The solid line represents the solidbody rotation at $(1-\mathrm{Ro})\left(r_{48} / R\right)$, and the dashed line represents the solid-body rotation at $(1+\mathrm{Ro})\left(r_{48} / R\right)$.

there is a discontinuity when we cross from the slow to the fast hemicylinder.

On the other hand, the bulk velocity corresponds to the points that are closer to $u_{\theta}=1$. Two different behaviors can be observed. For $\operatorname{Re}=4 \times 10^{3}$ the fastest state of the slow cylinder approximates the solid-body velocity $u_{\theta}\left(r_{48}\right)=0.96$ when we move from the base to the gap, and then increases to larger values when we approach the fast end. Meanwhile, for large Re this bulk velocity is constant whatever is the $z$-position, although slightly smaller than the velocity $u_{\theta}^{\text {solid }}(r)$, and approaching $\left(r_{48} / R\right) u_{\text {wall }}^{-}$when Ro increases. For $z=$ $8 \mathrm{~mm}$ and $z=40 \mathrm{~mm}$ a third state of the velocity appears between which it becomes significant when Re increases.

Figure 7 represents the behavior of the dimensionless azimuthal velocities $\bar{u}_{\theta}^{i}$ as a function of the axial position for different $\mathrm{Ro}$ and for $\mathrm{Re}=4 \times 10^{3}$ (top panels) and $\mathrm{Re}=$ $2 \times 10^{4}$ (bottom panels). The principal result presented is related to the evolution of the state which corresponds to the solid-body rotation. For small $\mathrm{Re}$ the velocity of this state progressively increases from a value below the average velocity $u_{\theta}^{\text {solid }}\left(r_{48}\right)$ when we are inside the slow cylinder to a value above the average when we are on the fast cylinder that rotates with a frequency $\Omega+\omega$. This increment of the velocity with the axial position decreases when $R e$ increases and tends to a real solid-body rotation value independent of $z$ for the highest $\mathrm{Re}$ (in accordance with the Taylor-Proudman theorem [25]). When the Re is low, the large Ro creates a high asymmetry along the cylinder which is reduced when Re increases. In other words, when Re is large, the boundary layer is thinner and the solid-body rotation is reached closer to the wall. On the other hand, the other states of the velocity field abruptly switch from below the solid-body rotation state in the left cylinder to above the solid-body rotation in the right cylinder. 

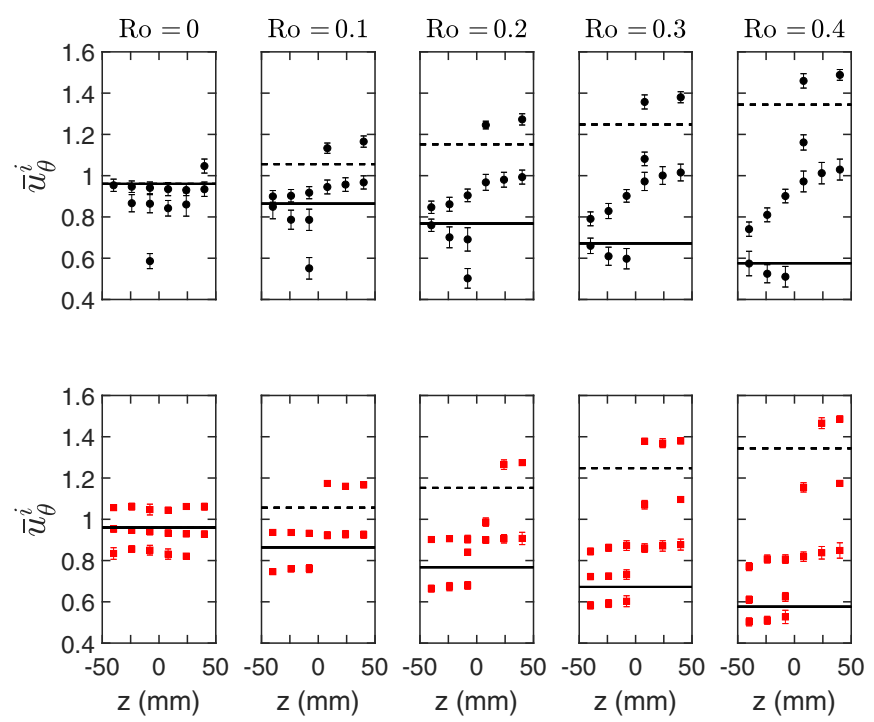

FIG. 7. Dimensionless azimuthal velocities $\bar{u}_{\theta}^{i}$ vs the axial position at $r=48 \mathrm{~mm}$ for different Ro and for $\mathrm{Re}=4 \times 10^{3}$ (top panels) and $\mathrm{Re}=2 \times 10^{4}$ (bottom panels). The solid line represents the solid-body rotation at $(1-\operatorname{Ro})\left(r_{48} / R\right)$, and the dashed line represents the solid-body rotation at $(1+\mathrm{Ro})\left(r_{48} / R\right)$.

This modulation of the velocity near the lateral boundary layer can also appear in other components. Here we present the radial evolution of the axial component $\left(v_{z}\right)$ for the same parameters used in Fig. 3. The axial velocity is measured each $0.5 \mathrm{~mm}$ using an acquisition time of $600 \mathrm{~s}$ per point in order to have a good statistics of this component due to its low value ( $\lesssim 1 \mathrm{~mm} / \mathrm{s})$. This velocity is nondimensionalized using $V$ and Ro, because this component appears when Ro $\neq$ $0\left[u_{z}(r, z, t) / \operatorname{Ro}=v_{z}(r, z, t) /(V \operatorname{Ro})=v_{z}(r, z, t) /(\omega R)\right]$. The mean of the axial component over the Ro, $U_{z}(r, z) / \operatorname{Ro}=$ $\left\langle u_{z}(r, z, t)\right\rangle / \mathrm{Ro}$, is presented in Fig. 8 averaging this mean each two measuring points (hollow black points in Fig. 8) in order to reduce the effect of the length of the experimental volume in the radial direction $(\sim 1.7 \mathrm{~mm})$. The dimensionless standard deviations of these points are $\sim 0.01$.

According to Ref. [28] the expected behavior of this component of the velocity field is a fluctuation over a null velocity. If we move from the wall to the interior of the flow this fluctuation in the boundary layer first points from the faster side to the slower side, then from the slower to the faster side and then it goes back again to the slower side creating a sandwich-like boundary layer like in the Stewartson's problem [24].

The experimental velocity (Fig. 8) is a little more complex. The main difference is that the experimental flow has a net mass transport across this radius $(z=40 \mathrm{~mm}, \theta=0)$. For an axisymmetric flow, this mass transport should be zero. As a first approach, we can assume that the experimental flow combines two different behaviors. The first one is related to the fluctuations described before [28], and the second one is related to a global circulation. This circulation is identified as a Kelvin mode in the cylindrical cavity [25], where the axial dependence of the correspondent $z$-component is a function of the azimuthal wave number. Our visualizations using Kalliroscope show a $m=1$ mode (stationary). Hence, the axial component of the velocity, $U_{z}$, is separated in these

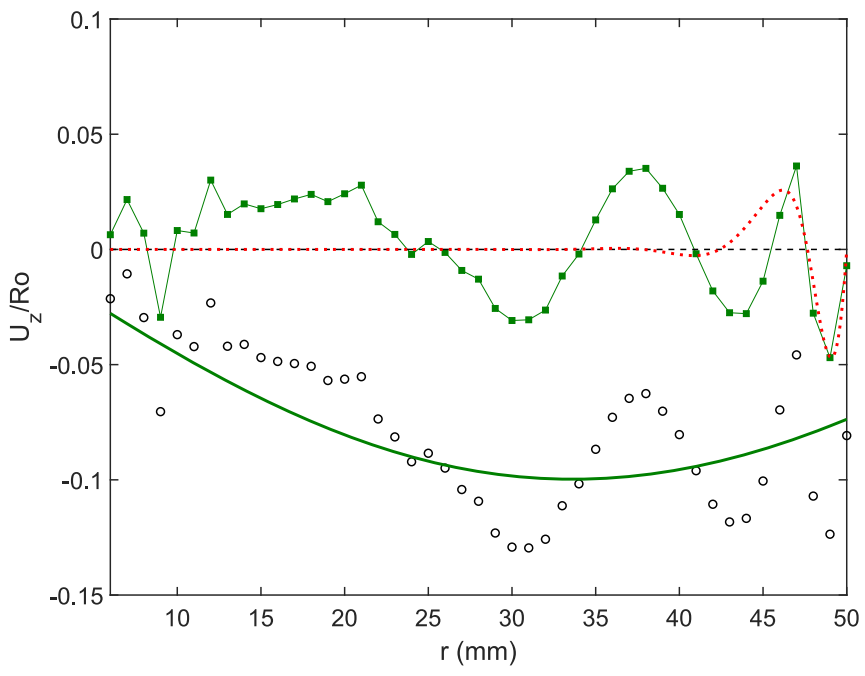

FIG. 8. Dimensionless axial velocity over Ro along a radius at $z=40 \mathrm{~mm}$ and $\theta=0$ for $\operatorname{Re}=2 \times 10^{4}$ and $\operatorname{Ro}=0.1$. Hollow black points are the mean axial experimental velocity, the thick green line corresponds to the Kelvin mode, and the green squares are the fluctuations over this mode. The red dotted line represents the theoretical profile using boundary layer theory [27], and the black dashed line represents the null velocity.

two contributions, and it can be expressed as $U_{z}=U_{z}^{\prime}+U_{z}^{K}$, where $U_{z}^{K}$ is the global Kelvin mode and $U_{z}^{\prime}$ are the fluctuations that do not produce a transport of mass across any radius, so $\int_{0}^{R} U_{z}^{\prime} r d r=\int_{0}^{R}\left(U_{z}-U_{z}^{K}\right) r d r=0$.

We suppose a global Kelvin mode in the form $U_{z}^{K}=$ $a J_{1}\left(\delta_{i} \frac{r}{R}\right)$ [25,30-32] where $a$ is the amplitude, $J_{1}(x)$ is the Bessel function of the first kind, and $\delta_{i}$ is the radial wave number. This radial wave number is solution of the Kelvin's dispersion relation $\delta_{i} J_{1}^{\prime}\left(\delta_{i}\right)+\sqrt{1+\frac{\delta_{i}^{2}}{k_{i}^{2}}} J_{1}\left(\delta_{i}\right)=0$ being $J_{1}^{\prime}(x)$ the $x$ derivative of the Bessel function and $k_{i}$ the axial wave number (see Ref. [25] for further details). For these calculations the corresponding wave numbers are obtained using dimensionless radius equal to 1 , and dimensionless height equal to $\Gamma$. With these values, and taking into account that the flow fills the whole container, $k_{i}$ has to be equal to $\pi / \Gamma$. Hence, using this axial wave number, the radial wave number can be obtained numerically from the previous dispersion relation using the bisectrix method. This mode is represented in Fig. 8 as a thick green continuous line, and the fluctuations over this mode are represented by green solid squares.

A careful check of our experimental setup revealed that there is a misalignment of the orientation of the shafts, and then the axes are not coaxial, this misalignment being $\theta_{\text {axis }} \lesssim$ $0.32^{\circ}$. This breaks the axial symmetry and provokes the global recirculation. This symmetry breaking is stationary on the laboratory reference frame and thus produces a stationary $m=1$ mode. All the dynamics present on the experiment can be altered by this fact, and this can explain why other modes with higher $m$ have not been observed. This global mode does not affect the rest of the results as there is not a nonlinear interaction among the global mode and the other phenomena found. 


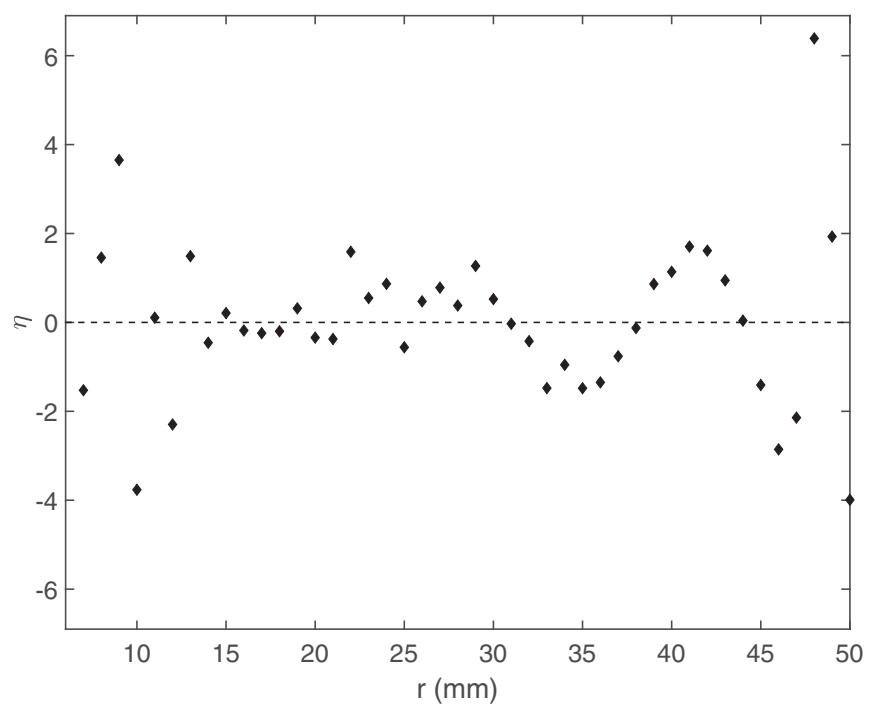

FIG. 9. Dimensionless azimuthal vorticity $\eta$ created by $U_{z}^{\prime}$ along a radius at $z=40 \mathrm{~mm}$ for $\mathrm{Re}=2 \times 10^{4}$ and $\mathrm{Ro}=0.1$. The black dashed line represents the null vorticity.

The frequency of this Kelvin mode measured in the rotating reference frame of the solid-body rotation is $-0.995 \Omega$, so we can assume that, in our case, the Kelvin mode is stationary in the laboratory reference frame, a result that corresponds to the experimental situation. Actually, a Kelvin mode perfectly stationary with a frequency $-\Omega$ is reached for an aspect ratio of $\Gamma=1.988$, slightly smaller than ours, but we are close enough to trigger this mode in our setup due to the misalignment. Finally, the value of the axial velocities are compatible with the axial flow due to the different pumping of the Ekman layers on both ends $U_{z} / \operatorname{Ro} \sim \operatorname{Re}^{-1 / 2} \sim 0.01$ [27].

These fluctuations recover the oscillating meridional flow first pointing from the faster side to the slower one, then going back and so on but, in the experimental case, these fluctuations are present all along the radius, not only in the boundary layer. We compare these values with the theoretical profile obtained using the boundary layer theory developed in Ref. [27] for $\operatorname{Re}=2 \times 10^{4}$ at $z=40 \mathrm{~mm}$ (red dotted line in Fig. 8). This boundary layer theory neglects nonlinear terms and uses Ro $\ll 1$. In order to compare the experimental fluctuations with the shape of the theoretical profile the same strategy used in Ref. [33] is applied here where the theoretical profile is rescaled to match the experimental result. Both theoretical and experimental axial velocities are similar near the wall, but they differ for $r \lesssim 46 \mathrm{~mm}$. These differences come from the strong nonlinearities and because our Ro cannot be considered a perturbation.

The dimensionless azimuthal vorticity $\eta$ created by the fluctuations $U_{z}^{\prime}$ is also computed and shown in Fig. 9 supposing that the effects of the radial velocity over this component of the vorticity are negligible at the measured z-position. This vorticity is quantitatively similar to the vorticity computed in Refs. [23,28] in their numerical simulations even taking into account that our parameters values for $\operatorname{Re}, \operatorname{Ro}$, and $\Gamma$ are different. $U_{z}^{\prime}$ also presents the shape described in Ref. [28] near the lateral wall, but the quantitative comparison is not presented because our parameters values differ from the ones used in that work.

\section{DISCUSSION}

According to the experimental data, two main phenomena have been found in the setup: the cylindrical wall boundary layer dynamics and the global recirculation mode.

The lateral wall boundary layer presents the classical "sandwich" structure predicted theoretically [24] and found numerically [28], where the two first peaks of the axial velocity from the cylindrical wall scale with $v^{1 / 3}$ and $v^{1 / 4}$, respectively.

But this boundary layer also has a complex dynamics near the cylindrical wall, evidenced by the jumps in the azimuthal component of the velocity field shown in Figs. 5-7. These jumps correspond to the branches of Fig. 3, so it can be noted that, depending on the Re and Ro, the dynamics can jump or not, and the number of states can be two or more. All jumps have a temporal behavior which supports the idea of rolls traveling near the wall (this temporal behavior is clearly seen in Fig. 4). Moreover, in many of these jumps one of the branches has a velocity which is higher than the highest velocity $(1+$ Ro $)$. This is possible because the velocity field alternates among states with different values, so the energy of the split-cylinder flow is not higher than the injected energy, i.e., if the mean velocity of each measurement is computed, it remains below $(1+$ Ro $)$.

Concerning the global mode, we can appreciate in the averaged flow of Fig. 2 that the solid-body rotation zone is tilted near the axis. This tilting can be characterized with the angle $\theta_{\text {flow }}$ formed between the contour line defined by $U_{\theta}=0$ and the rotation axis when it crosses the axis $(r=$ $0 \mathrm{~mm}$ ) in Fig. 2. When the radius is small, the effect of this misalignment becomes noticeable, and the velocity deviates from the solid-body rotation. This can also be observed in Fig. 3 for little radii.

The Kelvin modes described in Ref. [25] are obtained for the inviscid case and cannot explain this behavior [this theory predicts that $\left.U_{\theta}(r=0)=0\right]$. But, when viscosity is included, the flow becomes tilted (i.e., the line where $U_{\theta}=0$ forms an angle with the axis of rotation) as has been observed in precessing cylinders (e.g., see Refs. [30-32,34]). In those cases, the presence of a secondary angular velocity breaks the axial symmetry of the problem and a global mode is created. In our case we have a similar behavior: even in perfect corotation $(\mathrm{Ro}=0)$, the misalignment $\theta_{\text {axis }}=0.32^{\circ}$ breaks the axial symmetry and then a global mode is created in the velocity field with a tilting of $\theta_{\text {flow }} \sim 9^{\circ}$ as can be observed in Fig. 2.

Finally, we have observed that two columns appear near the axis of the cylinder as those shown in Fig. 10. These columns can remember the ones in Fig. 2 of Ref. [34], but we do not have a cylinder in precession. The origin of these columns can be different from the global mode found in the system, and they could appear even in an ideal split-cylinder flow. But, in this case, these columns had to be parallel to the axis due to the lack of a deviation angle between semicylinders. This represents a work under progress. 


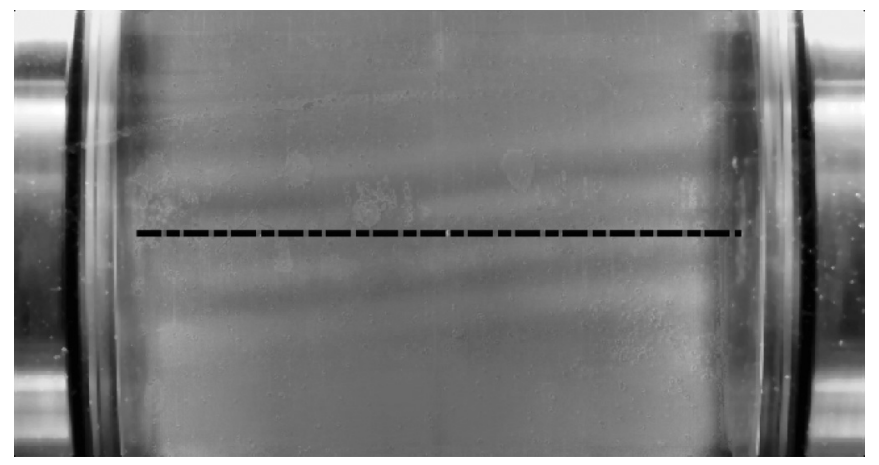

FIG. 10. Snapshot of the experimental setup using Kalliroscope for $\operatorname{Re}=2 \times 10^{4}$ and $\mathrm{Ro}=0.1$ for $r<0.63 R$. A median filter has been applied to eliminate the noise. The uneven illumination is removed averaging a series of snapshots that covers the timescales of the dynamics. Each snapshot is then divided by this average image to highlight the dynamical behavior.

\section{CONCLUSIONS}

The cylindrical wall boundary layer of the split-cylinder problem in corotation is studied experimentally and compared with theoretical and numerical results. Due to the high Re studied, the flow is essentially in solid-body rotation when $\mathrm{Ro}=0$. A secondary flow develops when $\mathrm{Ro} \neq 0$ driven by the difference of rotation velocities and restricted to the boundary layers.
The azimuthal velocity has a complex behavior inside this boundary layer consisting of regular alternative jumps among different values of this component of the velocity field. These alternations have been associated with waves that travel along with the cylindrical wall in the fast (respectively, slow) cylinder at its rotation frequency $\Omega+\omega$ (respectively, $\Omega-\omega$ ). On the other hand, a differential rotation breaks the reflection symmetry of the velocity field at the equatorial plane, but this symmetry can be recovered at larger background rotations (larger Re) as predicted by the Taylor-Proudman theorem. A "sandwich-like" structure has been found in the axial component that scales as previous results predict. But, in our experiment, the fluctuations of this component of the velocity field appear along the whole radius.

Finally, a stationary global mode (in the laboratory reference frame) with azimuthal number $m=1$ is found related to a little misalignment intrinsic to the current experimental setup. This behavior can be assimilated to results found in precession experiments. Due to this forced symmetry breaking, modes with higher $m$ cannot occur.

\section{ACKNOWLEDGMENTS}

We are grateful to Luis Fernando Urrea, our laboratory technician, for his assistance during the assembly and the adjustment of the experimental setup. This work has been supported by the Spanish Ministry of the Economy under Projects No. FIS2014-54101-P and No. FIS2017-83401-P. J.O.R.-G. is supported by a Ph.D. Grant from the "Asociación de Amigos de la Universidad de Navarra."
[1] E. Crespo del Arco, E. Serre, P. Bontoux, and B. Launder, Instability of Flows (MIT Press, Cambridge, MA, 2005), Vol. 6, pp. 141-195.

[2] G. K. Batchelor, J. Fluid Mech. 20, 645 (1964).

[3] D. W. Moore and P. G. Saffman, Proc. R. Soc. A 333, 491 (1973).

[4] C. del Pino, L. Parras, M. Felli, and R. Fernandez-Feria, Phys. Fluids 23, 13602 (2011).

[5] F. J. Blanco-Rodríguez, J. O. Rodríguez-García, L. Parras, and C. del Pino, Phys. Fluids 29, 64108 (2017).

[6] M. P. Escudier, Exp. Fluids 2, 189 (1984).

[7] J. M. Lopez, J. Fluid Mech. 221, 533 (1990).

[8] A. de la Torre and J. Burguete, Phys. Rev. Lett. 99, 054101 (2007).

[9] M. López-Caballero and J. Burguete, Phys. Rev. Lett. 110, 124501 (2013).

[10] C. Nore, L. Martin Witkowski, E. Foucault, J. Pécheux, O. Daube, and P. Le Quéré, Phys. Fluids 18, 054102 (2006).

[11] C. Nore, F. Moisy, and L. Quartier, Phys. Fluids 17, 064103 (2005).

[12] J. Burguete and A. de la Torre, Int. J. Bifurcation Chaos 19, 2695 (2009).

[13] O. Liot and J. Burguete, Phys. Rev. E 95, 013101 (2017).

[14] R. Monchaux, M. Berhanu, M. Bourgoin, M. Moulin, P. Odier, J.-F. Pinton, R. Volk, S. Fauve, N. Mordant, F. Pétrélis et al., Phys. Rev. Lett. 98, 044502 (2007).
[15] L. Marié, J. Burguete, F. Daviaud, and J. Léorat, Eur. Phys. J. B 33, 469 (2003).

[16] A. de la Torre, J. Burguete, and C. Pérez-García, Eur. Phys. J.: Spec. Top. 146, 313 (2007).

[17] A. Giesecke, F. Stefani, and J. Burguete, Phys. Rev. E 86, 066303 (2012).

[18] P. Huck, C. Bateson, R. Volk, A. Cartellier, M. Bourgoin, and A. Aliseda, J. Fluid Mech. 846, 1059 (2018).

[19] N. Machicoane, M. López-Caballero, L. Fiabane, J.-F. Pinton, M. Bourgoin, J. Burguete, and R. Volk, Phys. Rev. E 93, 023118 (2016).

[20] D. Dijkstra and G. J. F. van Heijst, J. Fluid Mech. 128, 123 (1983).

[21] J. E. Hart and S. Kittelman, Phys. Fluids 8, 692 (1996).

[22] J. M. Lopez and F. Marques, Phys. Fluids 22, 114109 (2010).

[23] J. M. Lopez and P. Gutierrez-Castillo, J. Fluid Mech. 800, 666 (2016).

[24] K. Stewartson, J. Fluid Mech. 3, 17 (1957).

[25] H. Greenspan, The Theory of Rotating Fluids, Cambridge Monographs on Mechanics (Cambridge University Press, Cambridge, 1968).

[26] L. M. Hocking, J. Fluid Mech. 12, 129 (1962).

[27] G. J. F. Van Heijst, J. Fluid Mech. 130, 1 (1983).

[28] P. Gutierrez-Castillo and J. M. Lopez, Eur. J. Mechanics B 52, 76 (2015). 
[29] J. V. Sengers and J. T. R. Watson, J. Phys. Chem. Ref. Data 15, 1291 (1986).

[30] P. Meunier, C. Eloy, R. Lagrange, and F. Nadal, J. Fluid Mech. 599, 405 (2008).

[31] R. Lagrange, C. Eloy, F. Nadal, and P. Meunier, Phys. Fluids 20, 081701 (2008).
[32] R. Lagrange, P. Meunier, F. Nadal, and C. Eloy, J. Fluid Mech. 666, 104 (2011).

[33] R. P. J. Kunnen, H. J. H. Clercx, and G. J. F. van Heijst, J. Fluid Mech. 727, 509 (2013).

[34] T. Albrecht, H. M. Blackburn, J. M. Lopez, R. Manasseh, and P. Meunier, J. Fluid Mech. 841, R3 (2018). 